\title{
L'animation « Pourkoikomandonkou »
}

Une visite originale de la Grande Galerie de l'Evolution pour les enfants de cinq à sept ans

Cora Cohen, Valérie Godard, Michel Roger et Yves Girault

\section{(2) OpenEdition}

1 Journals

Édition électronique

URL : http://journals.openedition.org/trema/2051

DOI : 10.4000/trema.2051

ISSN : 2107-0997

Éditeur

Faculté d'Éducation de l'université de Montpellier

Édition imprimée

Date de publication : 1 septembre 1996

Pagination : 111-122

ISSN : 1167-315X

\section{Référence électronique}

Cora Cohen, Valérie Godard, Michel Roger et Yves Girault, « L'animation « Pourkoikomandonkou » », Tréma [En ligne], 9-10 | 1996, mis en ligne le 18 septembre 2013, consulté le 01 mai 2019. URL : http:// journals.openedition.org/trema/2051 ; DOI : 10.4000/trema.2051

Ce document a été généré automatiquement le 1 mai 2019.

Trema 


\section{L'animation}

\section{«Pourkoikomandonkou »}

Une visite originale de la Grande Galerie de l'Evolution pour les enfants de cinq à sept ans

\section{Cora Cohen, Valérie Godard, Michel Roger et Yves Girault}

\section{La Grande Galerie de l'Évolution}

1 Inaugurée le 21 juin 1994, la Grande Galerie du Muséum National d'Histoire Naturelle offre la particularité de présenter ses collections en prenant comme base la réflexion scientifique sur la théorie de l'Évolution. L'exposition permanente se déploie en trois actes.

2 Le premier acte développe le thème de la diversité du vivant sur deux niveaux. Au niveau 0 , la diversité des organismes est évoquée à travers des milieux marins et le niveau 1 traite de la diversité des milieux terrestres. Les quatre milieux choisis pour illustrer ce thème sont : les forêts tropicales d'Amérique, la savane africaine, le désert saharien et les régions polaires.

3 Le deuxième acte approfondit la réflexion suscitée par le spectacle de la diversité. Il présente l'évolution des organismes vivants depuis la création de la Terre ainsi que l'évolution des recherches scientifiques à ce sujet (de la fin du XVIIe siècle à nos jours).

4 Enfin, les relations entre l'homme et la Nature constituent la dernière étape de la visite de la Grande Galerie. Ce troisième acte se déroule sur une échelle de temps réduite, d'environ 10000 ans, au cours desquels les activités humaines ont transformé l'évolution du monde vivant et causé des modifications rapides, profondes et parfois irréversibles de l'environnement.

5 Le premier acte de la Grande Galerie de l'Évolution fait l'objet de l'animation «Pourkoikomandonkou » proposée aux enfants de cinq à sept ans. Cette activité, basée autour d'un conte, aborde le thème de la diversité des organismes dans la diversité des milieux ${ }^{1}$. Il semble judicieux, au préalable, d'introduire l'étude de cette animation par une 
présentation des visites organisées autour de contes dans les musées. En effet, celles-ci suscitent certaines questions: comment peuvent être exploités les contes dans les musées ? Quels sont les principaux objectifs des visites-contes? Et plus particulièrement dans l'activité "Pourkoikomandonkou », quelles sont les délimitations opérées dans le savoir savant pour sensibiliser les jeunes visiteurs au concept de biodiversité ? Quels sont les apports possibles des différentes formes utilisées (conte et visite) dans cette animation? Ces formes sont-elles pertinentes pour percevoir et saisir la notion de biodiversité?

6 Une analyse des différentes fonctions du conte permettra de donner un premier aperçu des atouts et des limites des visites-contes et d'introduire l'évaluation entreprise sur l'animation « Pourkoikomandonkou ». Dans un premier temps, une analyse conceptuelle tentera de saisir le découpage qui a été opéré dans le savoir savant pour réaliser cette activité. Dans un deuxième temps, une analyse didactique se penchera sur le dispositif mis en place pour cette activité.

\section{Les atouts et les limites des visites-contes}

\subsection{Les différentes fonctions du conte}

7 Le conte possède trois fonctions principales ${ }^{2}$ : il favorise l'évasion, la transmission de la culture et le développement individuel de l'enfant.

8 La fonction d'évasion du conte compense la frustration causée par les limitations sociales et biologiques. P. Peju (1981) souligne, par exemple que «le conte offre l'occasion de voyages mentaux au cours desquels s'abolissent les frontières entre l'humain et le végétal, l'animal et le végétal, entre désirs et réalités ». Il satisfait ainsi le désir qu'ont les mortels de dépasser leur condition terrestre. De plus, J. E. Bencheikh (1988) souligne que «le conte offre un lieurefuge, un espace où peut se tenir une parole tenue ailleurs prisonnière ». Ainsi, il brise de puissants tabous sociaux par le truchement des acteurs mis en scène. Par exemple, selon B. Bettelheim (1976), Le Petit Chaperon Rouge parle des passions humaines, de l'avidité orale, de l'agressivité et des désirs sexuels de la puberté.

9 Par ailleurs, la fonction pédagogique du conte permet à chaque peuple de transmettre ses valeurs, sa généalogie, son histoire et sa culture. L'individu n'est alors plus seul, il se reconnait, il se sent relié à un groupe et inséré dans une histoire. De plus, le conte valide la culture traditionnelle et propose des règles de conduite en soulignant la nécessité d'un comportement social convenable.

10 Enfin, dans l'apprentissage de la maturité, le conte joue un rôle indispensable : tout en divertissant l'enfant, il l'éclaire sur lui-même et favorise le développement de sa personnalité en l'aidant à régler les problèmes psychologiques de la croissance. En effet, avant d'éprouver dans la vie réelle les épreuves, l'enfant s'y essaie volontiers dans l'imaginaire (sans contrainte, ni sanctions), et comme le souligne J. Michel (1976) « quelle que soit l'invraisemblance des situations, l'enfant trouve toujours dans le conte l'écho de sa propre affectivité, de ses désirs et de ses élans ». De plus, le conte aide l'enfant à donner un sens à la vie : il montre que l'homme doit lutter pour résoudre la permanence en lui du bien et du mal, en opposition constante; il rassure quant aux problèmes de l'existence. Comme l'écrit J. Thomassin (1991), le conte dit à l'enfant « rien n'est impossible, aucun obstacle n'est insurmontable ou incontournable ». Même si ce qui nous attend semble injuste, surprenant, 
inattendu, il faut l'affronter sans fuir. Ainsi, le conte met l'enfant en présence de ces « difficultés fondamentales de l'homme » (B. Bettelheim, 1976) que sont vieillissement et mort, sous une forme symbolique qui les rend supportables.

11 Ainsi, les trois principales fonctions du conte (l'évasion, la transmission de la culture, et le développement individuel de l'enfant) contribuent au développement physique, intellectuel et psychique des enfants. Il sera intéressant de vérifier si les visites-contes répondent elles aussi à ces mêmes objectifs.

\subsection{Les différentes fonctions du conte au service du musée ${ }^{3}$}

L'introduction du conte dans les musées peut paraître insolite. À quelles fins peut-il bien être utilisé ? Une analyse approfondie des trois principaux objectifs des visites-contes permettra peut-être de répondre à cette question. Ces trois objectifs que sont l'éveil à la sensibilité, une nouvelle approche des disciplines, l'éducation du regard, ont été soulevés par la majorité des responsables pédagogiques rencontrés.

visites-contes souhaitent, d'une part, procurer du plaisir aux enfants afin de les inciter à revenir au musée et d'autre part, les familiariser avec des œuvres d'art: peintures, sculptures, objets d'art d'époques, d'origines, de genres et de styles différents. Le Musée du Petit Palais ${ }^{4}$ organise, par exemple, des classes-découvertes sur le thème "les Créatures Fantastiques». Ce type de visite se déroule sur trois séances de deux heures. A chaque séance, la classe est accueillie dans le musée par une conteuse professionnelle qui a sélectionné préalablement plusieurs œuvres d'art en fonction du projet pédagogique de l'enseignant et devant chaque œuvre choisie, les enfants écoutent un conte différent. Les jeunes sont conduits dans le monde merveilleux et imaginaire de l'œuvre d'art. Cette approche poétique, tout en suscitant l'intérêt pour la langue et les jeux de langage, présente l'avantage d'éveiller chez l'enfant sa curiosité et sa sensibilité au patrimoine artistique. Elle peut ainsi être l'occasion d'aborder quelques notions du programme scolaire.

14 La richesse des collections des musées peut être à l'origine de la création de situations d'apprentissages soit comme source d'un travail d'expression (situation motivante entraînant prise de parole, argumentation, échange d'impressions, création plastique...) soit comme illustration vivante et enrichissante d'un point du programme. Ainsi, les disciplines littéraires sont développées par la connaissance de contes et de légendes, par le travail de l'imaginaire et de l'expression orale ou écrite (narrative ou poétique), par l'enrichissement du vocabulaire, etc. Le Musée du Petit Palais a ouvert, par exemple, ses «ateliers artistiques, imaginaires et plastiques » aux élèves des classes de Maternelle Grande Section, CP et CE1 des ZEP (Zone d'Éducation Prioritaire) dans l'optique d'améliorer leur maîtrise de la langue française. Ces ateliers artistiques, imaginaires et plastiques se déroulent en trois séances de deux heures. La première séance est une visite générale destinée à familiariser l'enfant avec le musée, son rôle, la variété des collections. Cette découverte alterne contes et éléments artistiques sur le sujet choisi. Elle se conduit avec la classe toute entière pour que les repères soient communs à tous. Lors des deux séances suivantes, le groupe est divisé en deux. L'un s'occupe de la re-création orale d'un conte, l'autre effectue un travail plastique en deux ou trois dimensions, les deux groupes étant nourris de tout ce qu'ils ont découvert ensemble lors de la première séance. Après l'animation, les deux groupes peuvent échanger leurs expériences différentes en classe. C'est l'occasion d'une réappropriation des informations par les enfants. Chaque groupe a 
donc un "patrimoine commun » (première séance) et une expérience complémentaire différente (conte ou travail plastique) qu'il faut communiquer à l'autre groupe. Ceci permet une valorisation du travail de chacun par rapport à l'autre et débouche sur une exploitation plus riche.

Les visites-contes peuvent permettre également d'aborder quelques chapitres du programme d'histoire. Chaque société crée ses propres œuvres d'art et ses propres contes, reflets de son histoire, de ses coutumes et de sa culture. Ainsi, les enfants découvrent la civilisation égyptienne au Musée du Louvre ${ }^{5}$ en écoutant une histoire de l'Antiquité, "Sinouhé », illustrée par les nombreuses œuvres d'art et outils de la vie quotidienne retrouvés dans les pyramides de Khéops, Képhren et Mykérinos à Guizeh. De même, le Musée Carnavalet ${ }^{6}$, cherchant à privilégier la rigueur historique, a créé des conférences-contées. Par exemple, la conférencière relate, sous forme de conte, la fabrication du berceau conçu pour l'enfant de Napoléon III en n'utilisant que des données véridiques. Ce type d'animation demande, bien sûr, une lourde préparation puisqu'elle nécessite une recherche historique et l'écriture du conte. Ainsi les visites-contes permettent aux enfants une approche des œuvres d'art et de leur civilisation par l'imaginaire et le rêve. Cependant, l'œuvre ne doit pas être réduite à une illustration d'un cours d'histoire ou de littérature.

Le défaut le plus répandu dans les visites proposées par les musées d'Art est de privilégier l'approche historique des œuvres sans prendre en compte l'approche esthétique comme le soulignait déjà en 1974, M.T. Gazeau. Or il importe de ne pas limiter l'œuvre à son seul aspect documentaire ou didactique: représentation d'un mode de vie, reflet d'une époque, mais bien de l'appréhender dans toute sa dimension esthétique, c'est-à-dire, dans tout ce qui en fait une œuvre d'art. Le rôle du musée ne serait-il pas d'« apprendre à voir » comme celui de l'école est d'« apprendre à lire »? Comme l'école, le musée a, en effet, un rôle essentiel dans un certain nombre d'apprentissages fondamentaux: éducation sensorielle, éducation tactile, surtout éducation du regard pour mieux appréhender les volumes, les compositions et les couleurs. Les visites-contes réunissent l'art du conte à l'Art. Par la méthode dite d'imprégnation, les enfants écoutent une histoire, installés devant une peinture, une sculpture, un objet d'art ou une tapisserie. Par le biais du conte, ils admirent le portrait de la jolie fille du meunier (portrait de Berthe Morisot) dont ils suivent les aventures devant un tableau de Breughel (œuvres exposées au Musée du Petit Palais). Ils frémissent devant les dragons qui ornent les plats du Musée Guimet ${ }^{7}$. Tout naturellement, ils regardent les œuvres d'art. Elles prennent pour eux une réalité et deviennent intéressantes, vivantes. Peu à peu ils apprécient leur beauté, leur diversité et ils aiment en parler. Ainsi, l'approche esthétique des œuvres, proposée à travers les visites-contes, stimule chez l'enfant sa propre créativité et éveille le désir de s'exprimer. En ce sens, elle contribue à la formation globale de la personnalité.

17 Cette étude illustre l'utilisation du conte dans les musées d'Art, mais comment cet outil pédagogique peut-il être exploité dans un musée scientifique? Il serait intéressant de vérifier si l'animation «Pourkoikomandonkou» organisée à la Grande Galerie de l'Évolution favorise, comme les visites précédemment présentées, l'observation et l'acquisition de connaissances tout en procurant du plaisir aux enfants. 


\section{L'animation « Pourkoikomandonkou »}

18 L'animation "Pourkoikomandonkou», est proposée aux groupes scolaires et particulièrement aux élèves du cycle II du primaire (5-7ans). Chaque animation d'une durée globale d'une heure, se compose de deux parties d'environ 25 minutes chacune :

- La première partie se déroule dans une salle conçue et aménagée pour les enfants. Ils y suivront un conte qui permettra d'introduire la visite dans la Grande Galerie de l'Évolution.

- La deuxième partie est une visite de quelques espaces de la nef de la Grande Galerie.

\subsection{Approche conceptuelle}

L'animation « Pourkoikomandonkou » a pour objectif général de sensibiliser les enfants à la notion de diversité des espèces dans la diversité des milieux. La première partie de cette animation a pour objectif spécifique de permettre à l'enfant de se construire une grille de lecture pour la visite qu'il fera dans la nef, en fonction des questions qu'on l'a incité à se poser.

Dans notre étude, lors de l'analyse des champs conceptuels référents ${ }^{8}$, nous avons mis en évidence différents concepts en jeu dans cette activité. Nous entendons par concept « une représentation symbolique (presque toujours verbale) qui se présente sous forme d'une dénomination ayant une signification générale, pour un individu ou un groupe d'individus, restituable sous forme d'énoncé(s), utilisée dans le fonctionnement de la pensée, ayant une signification générale pour un individu ou un groupe d'individus » (M. Roger, 1994). Les concepts retenus sont les suivants : écosystème, biocénose, biotope, biosphère, climat, végétation, et milieux. Tous ces concepts ne sont pas directement traités avec les enfants, mais étant tous imbriqués, ils sont forcément en jeu dans cette animation. C'est sur le concept de milieu que nous allons nous attarder ici, par l'intermédiaire des trois milieux présentés dans cette activité, la forêt tropicale, le désert saharien, et la savane africaine. Certaines caractéristiques de ces milieux ont été retenues pour cette animation.

21 La forêt tropicale se distingue par la prédominance des arbres. Elle est peuplée par une communauté d'êtres vivants possédant une structure et une organisation complexe. Ces forêts abritent plus de $90 \%$ des espèces animales et végétales de la planète. La température élevée et l'humidité de l'air y sont assez constantes. D'un point de vue écologique, la forêt est composée de trois niveaux principaux ; ainsi, les conditions de vie à la cime des arbres diffèrent considérablement de celles du sol de la forêt.

22 La savane africaine se caractérise par de larges plaines ouvertes parcourues par des milliers d'animaux. Il est à ajouter que les précipitations sont souvent irrégulières. Ce milieu se caractérise par une alternance de deux saisons, provoquant des migrations d'animaux.

Dans le désert saharien, la surface du sol se réchauffe considérablement le jour, alors que la nuit, elle se refroidit rapidement déterminant une chute importante des températures. Les animaux du désert se voient imposer, pour survivre, une série d'adaptations tant au niveau physiologique que comportemental, le problème primordial à résoudre étant celui de l'approvisionnement en eau. 

présentation des trois milieux. Un animal a été choisi pour représenter chacun des milieux : le lion pour la savane africaine, le tapir pour la forêt tropicale, et le dromadaire pour le désert saharien. Par la mise en correspondance des trois animaux et des milieux choisis, le questionnement devient possible. En effet, un problème va émerger, puisque les enfants vont découvrir, tout d'abord, que n'importe quel animal ne peut pas vivre dans n'importe quel milieu, mais aussi, que ce phénomène doit s'expliquer. Dans la nef, les enfants auront une certaine grille de lecture pour la visite, et c'est lors de celle-ci que le concept de biodiversité sera directement traité et «illustré ». Ainsi les objectifs énoncés pour cette activité pourront être atteints si, d'une part, le conte permet de faire émerger un problème chez les enfants quant aux liens qui existent entre l'animal et son milieu, et d'autre part si les visites sont pensées en fonction de l'axe « diversité des animaux dans la diversité des milieux ». Après l'analyse conceptuelle de la partie conte, nous pouvons penser que la mise en problème peut exister chez les enfants. Nous verrons plus loin ce qu'il en est quant à la conception et la réalisation des visites par les animateurs.

\subsection{Approche didactique}

Cette approche très synthétique va tenter de décrire, commenter, et analyser le dispositif didactique mis en place pour cette activité. L'animation "Pourkoikomandonkou » demande un travail d'observation et de verbalisation de la part des enfants tant dans la partie conte que dans la partie visite.

\subsubsection{Le conte} l'enfant, ainsi il pourra entrer dans l'immense espace de la Grande Galerie plus rassuré. intitulé «Comment Napi créa les hommes et les animaux ». Dans ce conte, Napi l'ami du soleil, va avec de l'argile créer des animaux pour leur donner la vie. Puis, avant de leur indiquer un endroit pour vivre, il leur donnera des noms. En dernier lieu, Napi crée l'homme et lui annonce son lieu de vie. Mais quelque temps après, les animaux qu'il a créés reviennent pour se plaindre, ils ne supportent pas l'endroit dans lequel ils ont été envoyés. Trois animaux sont incarnés par des élèves : le lion, le tapir et le dromadaire. Ces derniers interviennent directement et parlent à Napi pour lui expliquer les raisons de leur mécontentement, l'homme en fait autant. Napi finira alors par rétablir la situation. Ce conte sensibilise les enfants au fait que n'importe quel animal ne peut pas vivre dans n'importe quel milieu.

Les trois animaux (le lion, le tapir et le dromadaire) choisis pour le conte sont les représentants des trois milieux (la savane africaine, le désert saharien, ou la forêt tropicale) qui sont présentés dans la nef. Françoise Cordier (1980), psychologue, s'appuyant sur les travaux de Rosch (1976), a construit des gradients de prototypie pour cinq catégories sémantiques dont les animaux. Elle refuse l'équivalence psychologique de tous les éléments d'une catégorie, et développe l'idée selon laquelle chaque élément ne serait pas un aussi bon représentant de la catégorie. Françoise Cordier constate qu'en ce

Tréma, 9-10 | 1996 
qui concerne la catégorie "animal», les exemplaires les plus représentatifs sont fortement confondus avec la catégorie des mammifères. Dans le conte présenté à la Grande Galerie, les trois animaux choisis sont des mammifères. Le fait d'utiliser comme représentants des trois milieux trois mammifères, permet aux enfants de rester proches de leurs représentations, sans "subir » de décalages entre le savoir proposé et le savoir qu'ils possèdent. Ne serait-il donc pas intéressant d'introduire un animal non mammifère, et cela pour inciter les enfants à sortir des prototypes qu'ils ont construits?

Pour cette activité, l'animateur va devoir jouer trois rôles. Avant que le conte ne commence, l'animateur se présente aux enfants en tant que conteur d'histoire. Celui-ci porte une blouse orange qu'il garde jusqu'à la fin du conte. Pour faire vivre Napi, personnage principal du conte, l'animateur porte un masque sur son visage. L'animateur joue ici deux rôles bien définis. Un troisième rôle vient se greffer aux deux premiers dès la fin du conte, puisque l'animateur devient alors conférencier, et il accompagne les enfants clans la nef pour la visite. Par rapport aux costumes, ce qui permet de distinguer le conférencier du conteur est la blouse puisqu'à la fin du conte le conférencier la retire. Ce retrait de costume est-il suffisant pour que les enfants distinguent le conteur et le conférencier ? L'animateur s'est présenté en tant que conteur au début de l'activité, puis il présentera Napi, seul le conférencier ne se présente pas. Ne prend-on pas le risque de faire exister le conteur pendant toute l'activité, et même pendant le moment de la visite? Est-ce vraiment nécessaire que l'animateur se présente en tant que conteur? Un conteur est un être capable de faire jaillir un univers, et d'y accompagner son public. Ne retire-ton pas d'une certaine façon la magie de ce personnage en le présentant? Une autre discussion peut s'appuyer sur la cohabitation du conteur et de Napi. En effet, pendant le conte, trois milieux sont étudiés : la savane africaine, la forêt tropicale, et le désert saharien. Des tableaux illustrant ces paysages sont exposés devant les enfants pour qu'ils puissent les observer et en discuter. Il nous semble logique que ce ne soit pas Napi qui parle aux enfants de ces écosystèmes, comme cela a été fait. Napi incarne ce qui est imaginaire dans ce conte, il ne peut pas avoir une approche scientifique des milieux. Après évaluation, réflexion, et consultation, les concepteurs de cette activité ont apporté les modifications adaptées aux problèmes énoncés plus haut. Désormais, le conteur ne se présente plus aux enfants, et une mise en scène nouvelle permet à Napi de s'éclipser pour laisser au conférencier le soin de faire observer et décrire les écosystèmes par les enfants.

Pour des raisons esthétiques, les architectes ont décidé de ne pas représenter les milieux dans la nef, seuls les décors et la mise en scène du conte autorisent la rencontre entre l'enfant et les milieux de vie des animaux. Les animaux y sont symbolisés par des figurines aimantées que l'on peut disposer sur les tableaux. Les représentations des animaux ne posent pas de problèmes exceptées celles de l'homme. En effet, l'homme s'est installé partout, et selon le milieu, son apparence sera différente. L'homme de la forêt tropicale est à bord d'une barque, celui de la savane africaine conduit un véhicule tout terrain, et c'est un nomade qui vit dans le désert. À partir de ces figurines, même les hommes se différencient selon les milieux où ils vivent, par leur moyen de transport, mais aussi leurs habitudes vestimentaires. Dans les représentations qui sont données des hommes par les figurines, ne sommes-nous pas ici sur les éventuelles représentations des petits européens assistant à cette animation? Et quelle est la validité de ces représentations par rapport à la réalité?

Lors de cette animation, les enfants selon leur âge ne réagissent pas de la même façon. Par exemple, pendant le conte, les jeunes enfants n'interviennent pas au moment où Napi 
place les animaux dans les mauvais milieux. Par contre les enfants plus âgés réagissent immédiatement en affirmant que Napi s'est trompé, et ils lui indiquent les bonnes associations entre les animaux et les milieux. Même si les enfants connaissent ces associations, ils n'ont peut-être pas encore compris que les animaux et leurs milieux de vie étaient intimement liés. Que les enfants réagissent ou pas, les animateurs ont tendance à rester enfermés dans le scénario pour lequel ils ont été formés. Pourtant, il serait intéressant de prendre en compte les réactions des enfants, car elles sont révélatrices d'un certain savoir en place. Ici se pose la question des grandes variations qui existe à l'intérieur de la tranche d'âge choisie, mais aussi de la marge de liberté qui est laissée aux animateurs par rapport à la formation qu'ils ont reçue.

\subsubsection{La visite} l'intérieur de la nef qui montrent les différents milieux. La plupart des enfants étant habitués, bercés, nourris, d'images vidéo ont du mal à détacher leurs yeux de ces «machines à hypnotiser». La majorité des animateurs n'utilisent pas ces écrans et tentent de les fuir; Os expliquent leur choix par le fait que le musée propose des spécimens que les enfants ne pourront pas voir ailleurs, alors que ces images ne sont pas spécifiques au musée. Les documentaires proposés sont muets, et si les animateurs ne les commentent pas, les enfants verront défiler des images sans en saisir tout leur sens. Sur une visite qui dure environ trente minutes, il nous semble dommage de se servir des écrans, alors que de nombreux spécimens merveilleux ou étonnants sont présents. Après nos observations, nous pouvons affirmer qu'il est difficile pour les animateurs d'éviter les écrans, les enfants étant nombreux dans un espace où les passages sont fréquents. Il est alors difficile de trouver un endroit idéal près du spécimen à observer.

Cette animation étant axée autour du concept de biodiversité, il nous semble nécessaire de présenter aux enfants lors de la visite dans la nef deux ou trois milieux pour leur permettre de réaliser des comparaisons entre les animaux qui les peuplent, et ainsi, pour mieux appréhender la notion de biodiversité. Réduire la visite à un seul milieu poserait des problèmes pour atteindre les objectifs de cette activité. En effet, observer que les animaux de la savane sont très grands permet de comprendre le lien qui existe entre les milieux de vie et les espèces vivantes qui les peuplent, mais cela ne suffit pas, surtout pour de jeunes enfants. Ce concept prend toute son ampleur si, après cette première observation, les enfants se retrouvaient devant les animaux de la forêt tropicale et pouvaient les comparer à ce qu'ils avaient vu précédemment. Entre les différents milieux, ces comparaisons peuvent se faire autour de plusieurs axes : leur taille, leur couleur, leur pelage, leur forme et leur déplacement. Devant les animaux, lors de la visite, il parait 
indispensable de rappeler les caractéristiques des milieux, puisqu'ils ne sont pas représentés dans la nef.

Cette visite doit s'articuler autour du concept de biodiversité, les animateurs doivent donc organiser leurs commentaires en fonction de cet axe. Ainsi, ils illustrent les concepts en s'appuyant sur l'observation de certains spécimens choisis. Après avoir suivi de nombreuses visites, il nous est possible d'affirmer que les animateurs sont tentés de raconter certaines anecdotes autour de la façon de vivre de ces animaux. Mais ces anecdotes, même si les enfants en sont friands, ne doivent pas devenir le point central des commentaires.

Quand nous analysons les différentes visites réalisées dans la nef de la Grande Galerie, nous nous rendons compte très rapidement que certains animateurs ne mettent pas en jeu le concept de biodiversité dans cette activité. En effet, ils construisent leurs visites en faisant appel à des notions comme celles d'herbivore, de Carnivore ou encore de chaîne alimentaire, sans traiter des concepts mis en évidence lors de l'analyse conceptuelle. Ainsi dans ce cas, les objectifs de l'activité ne pourront pas être complètement atteints. Les animateurs utilisent les éléments mis à leur disposition dans la nef sans toujours donner une priorité aux concepts choisis. Devant la richesse de la nef, les animateurs peuvent être tentés, comme nous l'avons vu lors de nos observations, de construire une visite en fonction des interrogations des enfants, de ce qui les intéresse, sans garder en tête les objectifs de cette activité.

Le personnage Napi.

Pour conter l'histoire de Napi, l'ami du soleil, au enfants, l'animateur se glisse dans la peau de son personnage en enfilant ce masque.

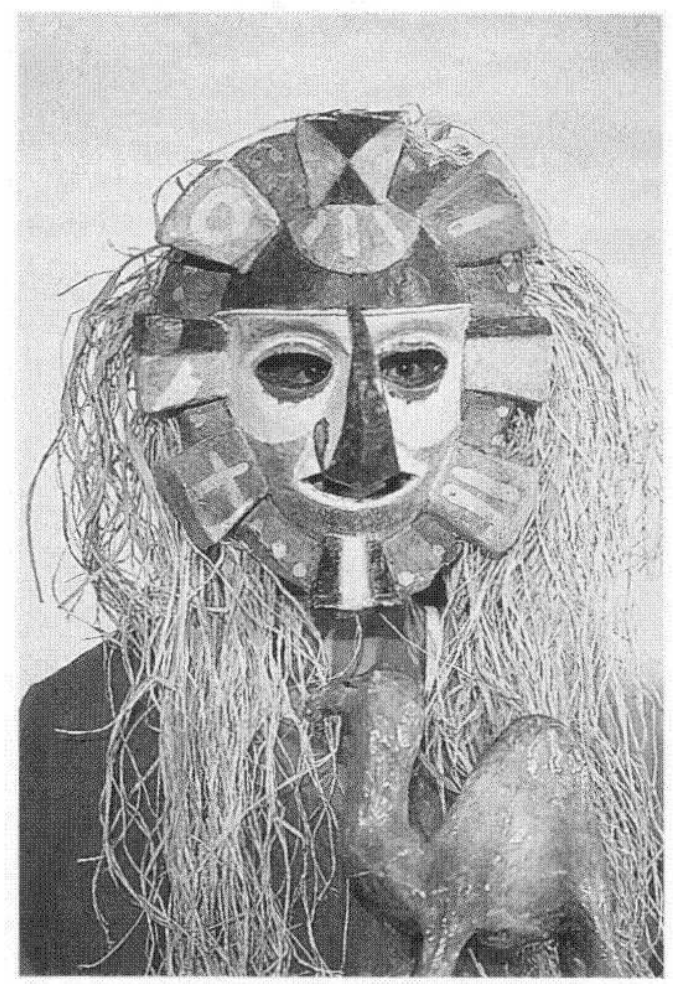

Photo : LAFAITE P., Muséum National d'Histoire Naturelle, Grande Galerie de l'Évolution, Copyright : 1996. 


\section{Conclusion}

Les deux formes utilisées pour cette activité (le conte et la visite) sont très complémentaires. En effet, une introduction sous forme de conte peut permettre si elle est bien pensée, et c'est le cas ici, de rendre la visite plus profitable puisqu'elle répond aux questions auxquelles les enfants viennent d'être confrontés. De plus, le côté imaginaire du conte rend l'activité plus plaisante aux jeunes enfants, et leur permet, dans un langage qu'ils connaissent bien, d'aborder des concepts ardus.

Dans l'activité « Pourkoikomandonkou », c'est dans la coexistence et dans l'enchaînement des deux parties qui la composent que certains problèmes se posent. En effet, cette activité subit une rupture tant au niveau didactique (passage de la fome conte à celle de visite) que conceptuel. Lit rupture didactique ne nous semble pas être un problème, au contraire, puisqu'elle semble assurer le passage entre le monde imaginaire du conte et celui « plus réel » de la nef et des animaux naturalisés. Par contre la rupture conceptuelle se révèle gênante puisqu'elle est un obstacle à l'atteinte des objectifs. Notre analyse a révélé que la plupart des visites réalisées par les animateurs peuvent être en décalage par rapport aux objectifs de cette activité. Nous pensons que ce problème peut être mis en relation avec la formation des animateurs, ou la part d'implicite qui existe dans chacune des actions de formation. Pour que l'activité «Pourkoikomandonkou » puisse être cohérente du point de vue conceptuel, il nous semble nécessaire de repenser certaines visites proposées aujourd'hui dans la Grande Galerie de l'Évolution. Après notre étude des modifications ont été apportées. Il serait intéressant d'établir une nouvelle évaluation pour en apprécier les résultats.

Quelques réflexions peuvent émerger en rapport avec la formation des animateurs. Nous avons pu remarquer que la partie pour laquelle les animateurs avaient reçu une formation qui leur offrait peu de marge de liberté, ne posait pas de problème par rapport aux objectifs fixés. Par contre, les animateurs semblent être gênés par l'inévitable problème de l'habitude. Ainsi, ils peuvent en arriver à réciter le conte plutôt qu'à le vivre. Les animateurs étaient plus libres par rapport à la conception de leurs visites, mais cette situation a entraîné une certain décalage par rapport aux objectifs. La formation est une étape délicate, cette animation en est un excellent exemple.

Cette recherche devrait se poursuivre par une étude des publics, des éventuels acquis engendrés par cette activité, et des modifications de leurs représentations.

\section{BIBLIOGRAPHIE}

BEANI L. \& DESSI F., Les savanes africaines. Paris, Larousse, 128 p., 1985.

BENCHEIKH J.E., Les « mille et une nuits » ou la parole prisonnière, Paris, Gallimard, Collection la bibliothèque des idées, 233 p., 1988.

BETTELHEIM B., Psychanalyse des contes de fées, Paris, Laffont, 320 p., 1976. 
CATALISANO A. \& MASSA B., Le désert saharien, Paris, Larousse, 128 p., 1986.

OUVRAGE COLLECTIF, Mille ans de contes d'animaux, "Comment Napi créa l'homme et les animaux ", Saint-Armand-Montron, Éditions Milan, 530 p., 1994.

COHEN C, L'animation Pourkoikomandonkou à la Grande Galerie de l'évolution, mémoire de maîtrise sous la direction de M. ROGER, 106 p., 1995.

CORDIER F., « La construction des gradients de prototypie pour cinq catégories sémantiques », in Psychologie française, Vol. 25, №3-4, 1980, p. 211-219.

DANSEREAU P., « Biogéographie », in Encyclopédia Universalis, Vol. 3, 1984, p. 632-640.

DOSSENBACH H., Vivre en forêt tropicale, Zurich, Suisse, Éditions Silva, 168 p., 1990.

GALOUX A. \& BOURNERIAS M., « Forêt », in Encyclopédia Universalis, Vol. 7, 1984, p. 1164-1179.

GAZEAU M.T., L'enfant et le musée, Paris. Éditions ouvrière. 156 p., 1984.

GENEST H. \& PETTER F., « Désert », in Encyclopédia Universalis. Vol. 5, 1984, p. 1177-1183.

GODARD V., Le conte : Vers une nouvelle exploration des musées parisien, Mémoire de DESS, sous la direction d'Y. Girault, 68 p., 1995.

MICHEL J., L'imaginaire de l'enfant, Paris, Nathan, 196 p., 1976.

PEJU P., La Petite Fille dans la Forêt des Contes, Paris, Laffont, 182 p., 1981.

ROGER M., « Champs conceptuels », in Principes des Actions Didactiques. Document de travail, UFR de Sciences de l'Éducation, Université René Descartes, 1994.

ROSCH E., «Classifications d'objets du monde réel : origines et représentations dans la cognition », in Bulletin de psychologie, $N^{\circ}$ Spécial : la mémoire sémantique. 1976, p. 242-249

THOMASSAINT J., Conte et (ré)éducation. Lyon, Éditions Chronique sociale, collection l'Essentiel, 99 p., 1991.

\section{NOTES}

1. Cette animation a été conçue par Françoise Robin, sous la responsabilité scientifique d'Yves Girault. Victor Serfaty et Patrick Michel ont créé les décors.

2. Ces trois fonctions sont les plus fréquemment citées par les cinq auteurs dont les écrits ont servi de base à la rédaction de cette partie (P. Peju, J.E. Bencheikh. B. Bettelheim. J. Michel, J. Thomassin).

3. 14 musées parisiens sur 61 proposent des animations autour des contes. Les faits rapportés ici sont issus d'entretiens réalisés par Valérie Godard avec les responsables pédagogiques de 10 musées parisiens (choix effectué en fonction de la disponibilité des responsables).

4. Extrait d'un entretien réalisé avec $\mathrm{M}^{\mathrm{me}}$ Masquelier-Boucher, responsable du service pédagogique du Petit Palais.

5. Extrait d'un entretien réalisé avec M. Soulié, responsable du service pédagogique du Musée du Louvre.

6. Extrait d'un entretien réalisé avec $M^{\mathrm{me}}$ Grégoire, responsable du service pédagogique du Musée Carcavalet.

7. Extrait d'un entretien réalisé avec $\mathrm{M}^{\text {me }}$ Fouillet, responsable du service pédagogique du Musée Guimet.

8. Le champ conceptuel référent est défini par Michel Roger (1994) comme suit: "Ensemble d'éléments de connaissances (concepts, opérateurs, règles et principes) servant de base 
référentielle à un champ conceptuel délimité par une (ou une suite de) situation(s) didactique(s) liée(s) à un programme ou à un objectif général. »

9. La formation pour le conte a été assurée pour une grande part par un marionnettiste : Alain Recoing.

\section{RÉSUMÉS}

Depuis une dizaine d'années, les musées organisent de plus en plus d'animations autour de contes pour faciliter les visites du jeune public tout en lui procurant du plaisir. Mais sont-elles nécessairement mieux adaptées aux enfants qu'une visite guidée traditionnelle et favorisentelles l'acquisition de connaissances? L'animation « Pourkoikomandonkou » organisée à la Grande Galerie de l'Évolution a été choisie pour servir de cadre à notre étude.

For ten years, museums have been staging more and more shows built around the telling of a story, in order to facilitate museum visits for the very young while at the came time entertaining them. But, are these visits necessarily any better suited to children than a conventional guided tour would be, and do they promote the acquisition of knowledge ? The "Pourkoikomandonkou" show, staged at the "Grande Galerie de l'Évolution" is chosen to serve as the context of our study.

\section{INDEX}

Keywords : biodiversity, climate, ecosystem, guided tours through storytelling, vegetation

Mots-clés : biodiversité, climat, écosystème, végétation, visite-conte

\section{AUTEURS}

\section{CORA COHEN}

Université René Descartes - Paris V, UFR de sciences de l'éducation

\section{VALÉRIE GODARD}

Muséum National d'Histoire Naturelle et université de Nice Sophia-Antipolis

\section{MICHEL ROGER}

Université René Descartes - Paris V, UFR de sciences de l'éducation

\section{YVES GIRAULT}

Muséum National d'Histoire Naturelle et université de Nice Sophia-Antipolis 